\title{
The Relationship Between Physical Activity and Premenstrual Syndrome in Female College Students
}

\author{
Kyoto University \\ Rika Kawabe \\ Kyoto University \\ Saori Morino \\ Osaka Prefecture University \\ Momoko Nagai-Tanima \\ Kyoto University \\ Tomoki Aoyama \\ Kyoto University \\ Kohei Mukaiyama \\ Kyoto University \\ Yuki Shinohara \\ Kyoto University \\ Hiroki Shimizu \\ Kyoto University \\ Kanako Shimoura \\ Kyoto University \\ Masaya Kato \\ Kyoto University
}

Chang Yu Chen ( $\square$ ccy0705@outlook.com )

\section{Research Article}

Keywords: Premenstrual syndrome, International Physical Activity Questionnaire, physical activity, woman's health

Posted Date: January 18th, 2022

DOI: https://doi.org/10.21203/rs.3.rs-1248368/v1

License: @ (i) This work is licensed under a Creative Commons Attribution 4.0 International License. Read Full License 


\section{Abstract \\ Background}

In recent years, moderate physical activity has attracted the attention of experts and women as a way to cope with premenstrual syndrome (PMS). Studies investigated the effects of exercise on PMS, but only a few reports focused on the relationship between physical activity, which included daily movement and exercise, and PMS. Therefore, the present study investigated the relationship between the amount of daily physical activity and PMS symptoms in sexually mature women.

\section{Methods}

A total of 381 female university students in Japan were surveyed using a paper or web-based questionnaire with the same content. The questionnaire consisted of basic information, PMS symptoms, and physical activity based on the International Physical Activity Questionnaire (IPAQ). Participants were divided into two groups ( $\geq 3000$ The Metabolic Equivalent of Task(MET)s/week and < 3000 METs/week) based on their total physical activity as calculated using the IPAQ guidelines. The two groups were then compared in terms of the severity of their PMS physical and psychological symptoms as calculated based on the American College of Obstetricians and Gynecologists' PMS diagnostic criteria. The Wilcoxon's rank-sum test was used for statistical analyses. We then divided the participants based on the presence or absence of each symptom using the Chi-square test. The significance level was set at $<5 \%$.

\section{Results}

Those with total physical activity of 3000 METs or more had lower total PMS symptom scores $(p<0.01)$, physical symptom scores $(p=0.01)$, and psychological symptom scores $(p=0.01)$ compared with those with total physical activity of $<3000$ METs/week.

\section{Conclusion}

These results suggest that people with moderate physical activity ( $\geq 3000 \mathrm{METs} /$ week) in daily life have milder symptoms of PMS.

\section{Background}

Premenstrual syndrome (PMS) is characterised by emotional, behavioural, and physical symptoms that occur during the late luteal phase of the menstrual cycle. It terminates after the onset of menstruation. Premenstrual dysphoric disorder (PMDD) is a more severe condition that includes functional impairment and disruption of personal relationships, such as depression [1][2]. There is abundant clinical research on perimenstrual symptoms, which include PMS and menstrual symptoms. The results of a study revealed that the frequency of PMS was relatively high (80 - 90\%) [3]. Other studies reported that 5-8\% of reproductiveaged women exhibited moderate to severe PMS symptoms that interfered with their daily activities [4].PMS may be caused by a variety of factors, mainly including sex hormones, autonomic nervous system, psychological factors, and lifestyle (sleep, diet, exercise, stress, and personal preferences) [5]. Many women prefer non-medical treatment options for PMS. This is mainly because they want to avoid the side effects, contraindications to drug treatments, and the high cost of treatment [4][6]. Current recommendations for coping with PMS include exercise [7] and dietary management [8]. These lifestyle changes are often cited as a way of dealing with PMS and are considered important non-pharmacological treatments that can be adjusted accordingly. However, there is a lack of comprehensive research on the relationship between lifestyle and PMS symptoms.

Several studies showed that exercise could improve PMS symptoms. These included aerobic exercises, such as swimming exercises [9]. Dahnavi et al. created an eight-week aerobic exercise plan [10]. The intensity was determined using the heart rate, 
which should be within 120-150 beats per minute (bpm) after exercise. Kamalifard et al. created a six-week yoga exercise regimen [11]. These two studies focused on exercises of different kinds and intensities.

Recently, physical activity (PA) and its ability to prevent menstruation-related symptoms have attracted the attention of experts and women [12]. PA is an activity that involves bodily movements resulting in energy expenditure. Only a few reports focused on the relationship between PMS and the amount of PA, including exercise and movement in daily life. One study examined PA's self-report measures using questionnaires, diaries, and brief logs [13]. However, only a few reports measured the regular PA in the daily lives of women in their childbearing age [14]. This vital information can provide a more detailed interpretation of the results.

The International Physical Activity Questionnaire (IPAQ) is the most representative questionnaire for assessing PA. Several studies reported the use of the IPAQ to measure PA $[15,16]$. IPAQ can be applied to participants with a widely varied age. In the IPAQ guidelines, a separate category labelled 'high' can be computed to describe higher levels of PA participation [17]. The criteria for classification as 'high' are seven or more days of any combination of walking, moderate-intensity, or vigorousintensity activities achieving a minimum total PA of at least 3000 MET-minutes/week.

Most of the studies [18] encouraged the employment of regular, moderate-intensity aerobic exercise[19] as a potential intervention for preventing PMS. Moderate-intensity aerobic exercises correspond to a high PA in daily life. Thus, we hypothesised that PMS symptoms were milder in people with a high PA. To test this hypothesis, we used the IPAQ to calculate the daily PA of participants. We then examined the relationship between PMS and daily PA in women of childbearing age. The purpose of this study was to investigate the relationship between PMS symptoms and PA levels.

\section{Methods}

This cross-sectional study (prospective questionnaire survey) designed to determine the relationship between PA and PMS symptoms was conducted following the Helsinki Declaration of 1975, as revised in 1983. The participants received information regarding our research and provided written or verbal consent after understanding the study protocol.

The present study was conducted in accordance with the guidelines of the Declaration of Helsinki and its amendments. This study was approved by the Kyoto University Graduate School and Faculty of Medicine and Kyoto University Hospital Ethics Committee (approval number: R1442). All information regarding participant data remains confidential. The participants' responses were anonymous.

\section{Participants}

In this study, 427 women consented to participate. This study was conducted in Japan between August and September 2016. The participants included in this study were a convenience sample consisting of undergraduate and graduate female students. The inclusion criteria were as follows: affiliated with a university in Japan, literate in Japanese so as to read and understand the proposed survey, potentially reproductive, and consented to participate. Those who were excluded from the study were menopausal women, those with current depression, anxiety, or any other psychiatric disorder previously diagnosed, those who received hormonal therapy, those who experienced a traumatic life event (widowhood, death of a close friend or relative, and imprisonment) before or during the study period, and those who were not due to drug therapy, hormonal internal use, or drugs.

\section{Questionnaire}

Our survey was administered via a paper or a web-based questionnaire with the same content. The questionnaire included basic information such as age, height, weight, menstrual conditions, levels of PMS symptoms, and PA.

\section{PMS}

PMS symptoms were referred to as premenstrual dysphoric disorder (PMDD) (An additional movie file shows this in more detail [see Additional file 1]) in aacordance to the American Psychiatric Association, 5th edition of the Diagnostic and Statistical 
Manual of Mental Disorders [20] and as PMS symptoms in the American College of Obstetrics and Gynecology guidelines (Additional file 2). We identified the symptoms among our participants by adding them in the questionnaire. We based our questionnaire on a previous study [21]. The symptoms we added were 'Lower abdominal pain', 'Increased appetite', 'Easily fatigued and feeling listless', 'Low back pain', 'Sleepiness', 'Decreasing concentration', and 'Fatigue or lack of energy Overeating'. The total number of PMS items in the questionnaire was 18. (Additional file 3).

To establish a diagnosis of PMS based on previous guidelines [22], we included a question regarding the presence of the symptoms in the past three menstrual cycles and whether at least one of the physical or psychological symptoms were observed 5 days prior to menstruation. The participants answered a questionnaire based on self-review. In this study, among all the items regarding symptoms, participants who checked as 'No problem but symptomatic' or 'Problematic' for one or more items were diagnosed with PMS.

All symptoms were separated into three levels. The 'problematic symptoms' corresponded to symptoms interfering with activities of daily living. 'No problem but symptomatic' corresponded to symptoms not interfering with activities of daily living. The final category, the 'no symptom', corresponded to the lack of symptoms.

The total score was calculated according to the following: no symptoms, 0 points; no problem but symptomatic, 1 point; and problematic symptoms, 2 points (Additional file 2).

For each symptom, we divided the patients into two groups: participants with and without symptoms.

PA

We used the short version of the IPAQ to determine the participants' daily PA. The IPAQ was developed as an instrument for cross-national monitoring of PA and inactivity. The IPAQ instruments have acceptable measurement properties, which are at least as good as other established self-reports. Moreover, they have reasonable measurement properties for monitoring the levels of PA among a population consisting of 18-65 years old adults with diverse backgrounds [23].

We asked about participants' moderate intensity active time, vigorous-intensity active time, and walking time. The participants' METs were then calculated according to the IPAQ guidelines. These were calculated as follows:

$$
\begin{gathered}
\text { WalkingMET }-\frac{\text { minutes }}{\text { week }}=3.3 * \text { walkingminutes } * \text { walkingdays } \\
\text { ModerateMET }-\frac{\text { minutes }}{\text { week }}=4.0 * \text { moderate }- \text { intensityactivityminutes } * \text { moderatedays } \\
\text { VigorousMET }-\frac{\text { min }}{\text { week }}=8.0 * \text { vigorous }- \text { intensityactivityminutes } * \text { vigorous }- \text { intensityday }
\end{gathered}
$$

TotalphysicalactivityMET - minutes $/$ week $=$ sumofwalking + moderate + vigorousMET - minutes $/$ weekscores

\section{Statistical analysis}

The PA data were divided into two groups according to the total metabolic equivalent of task (MET) in a week, which was defined by IPAQ as > 3000 MET-minutes/week and < 3000 MET-minutes/week). The two groups' severity scores for each of the PMS physical and psychological symptoms calculated based on the questionnaire were compared. Associations with characteristics were assessed using the Wilcoxon rank-sum test. We then divided the participants based on the presence or absence of each symptom using the chi-square test for comparison in terms of the IPAQ. The significance level was set at $<5 \%$. All analyses were conducted using the JMP Pro 14.0 (SAS Institute, Cary, NC, USA).

\section{Results}


The questionnaire was completed by a total of 381 female university students (89.22\%). The participants' characteristics are described in Table 1 and Table 2 (mean \pm SD, age: $20.4 \pm 1.2$ years; height: $159.5 \pm 5.6 \mathrm{~cm}$; weight: $51.7 \pm 5.8 \mathrm{~kg}$; Body Mass Indes(BMI): $20.27 \pm 1.78)$. Of these, $76.3 \%$ had regular menstrual cycles (25-38 days), while $70.87 \%$ students had a PMS.

Table 1

Basic information of participants

\begin{tabular}{|ll|}
\hline \multicolumn{2}{|c|}{ Botal $\mathrm{n}=\mathbf{3 8 1}$} \\
\hline Age $(\mathrm{y})$ & $20.44 \pm 1.23$ \\
\hline Height $(\mathrm{cm})$ & $159.57 \pm 5.58$ \\
\hline Weight $(\mathrm{kg})$ & $51.67 \pm 5.75$ \\
\hline BMI $\left(\mathrm{kg} / \mathrm{m}^{2}\right)$ & $20.27 \pm 1.78$ \\
\hline Total PMS score & $8.84 \pm 6.89$ \\
\hline Total physical activity (MET-minutes/week) & $4829.09 \pm 4537.48$ \\
\hline${ }^{*} p<.05$ & \\
\hline
\end{tabular}

Table 2

Menstrual information of participants

\begin{tabular}{|ll|}
\hline \multicolumn{2}{|l|}{ Regular menstrual cycles } \\
\hline Yes & $291(76.3)$ \\
\hline No & $90(23.6)$ \\
\hline PMS & \\
\hline Yes & $344(90.2)$ \\
\hline No & $37(9.7)$ \\
\hline
\end{tabular}

The results are shown in Table 3, the participants were divided into two groups based on their total PA. Those with total PA of 3000 METs/week or more had lower total PMS symptom scores $(p<0.01)$, physical symptom scores $(p=0.01)$, and psychological symptom scores $(p=0.01)$.

Table 3

The Wilcoxon's rank-sum test of group divided based on those who met at least 3000 METs and score of PMS symptoms

\begin{tabular}{|lllll|}
\hline \multicolumn{5}{|c|}{ Total physical activity (MET-minutes/week) } \\
\hline & $\geq 3000(\mathrm{n}=200)$ & $<3000(\mathrm{n}=181)$ & $\mathrm{z}$ & $p$ value \\
\hline Physical symptoms & 4.94 & 5.79 & 2.66 & $0.01^{\star}$ \\
\hline Psychological symptoms & 3.21 & 3.99 & 2.47 & $0.01^{\star}$ \\
\hline PMS symptoms & 8.16 & 9.78 & 2.88 & $0.00^{\star}$ \\
\hline${ }^{*} p<.05$ & & & & \\
\hline
\end{tabular}

We observed the effects of 3000 METs/week, depending on the presence or absence of each PMS symptom. As Table 4, the ratio of participants who with more than 3000 METs/week have no these symptoms is high: 'sleepiness', 'acne', 'feeling depressed', 'decreasing concentration', 'easily fatigued and feeling listless'. 
Table 4

The ratio of those with symptoms in the group divided based on the total METs

\begin{tabular}{|llll|}
\hline \multicolumn{4}{|c|}{ Total physical activity (MET-minutes/week) } \\
\hline With symptoms & $\geq 3000(\mathrm{n}=200)$ & $<3000(\mathrm{n}=181)$ & p value \\
\hline Sleepiness & $103(51.5)$ & $124(68.51)$ & $<0.01$ \\
\hline Acne & $61(30.5)$ & $80(44.20)$ & $<0.01$ \\
\hline Feeling depressed & $84(42.00)$ & $95(52.49)$ & 0.04 \\
\hline Decreasing concentration & $66(33.00)$ & $87(48.07)$ & $<0.01$ \\
\hline Easily fatigued and feeling listless & $84(42.00)$ & $96(53.04)$ & 0.03 \\
\hline $\mathrm{n}$ (row\%) & & & \\
\hline
\end{tabular}

Chi-square test

\section{Discussion}

In this study, we found a relationship between regular PA and PMS symptoms. This study clarified two results. First, people with a PA of $3000 \mathrm{METs}$ /week or more had lower scores for psychological and physical symptoms. Second, the ratio of participants who had more than $3000 \mathrm{MET}$ / week had no symptoms.

PMS is one of the most common problems among women. Moreover, PMS can interfere with activities of daily living. Thus, finding a way to prevent or treat PMS is an important health and research priority. In our study, we found that over $90 \%$ of women reported at least one symptom of PMS. This findings was similar to a study that found that $95 \%$ of participating women aged 18-24 years experienced at least one PMS symptom.[24]

Our first conclusion was that people whose PA is more than $3000 \mathrm{METs}$ /week had lower scores in psychological and physical symptoms. This conclusion complemented Koushkies et al.'s finding, which showed an association between an increased PA and a significant reduction in PMS symptoms [25]. However, Kroll-Desrosiers et al.'s study showed no evidence of an association between PA and either the presence of PMS or premenstrual symptom severity [14]. The difference must have been due to the difference in method. Kroll-Desrosiers et al.'s examined PA by continuous METs per week and analysed the data by dividing the group by tertiles of PA. Higher levels of participation were characterised using the IPAQ, which defined vigorousintensity activity 3000 MET-minutes/week or more. Furthermore, we used 3000 MET-minutes/week because this number approximated the recommended levels (150 minutes of moderate-intensity physical activity per week) of PA after calculation [26][27].

Regarding the second conclusion, the ratio of participants who had more than $3000 \mathrm{METs} /$ week had no symptoms. The symptoms we found in this study were 'sleepiness', 'acne', 'feeling depressed', 'decreasing concentration', and 'easily fatigued and feeling listless'. Some studies explained this phenomenon. Wilmore et al. showed that regular PA had many benefits, including reduction of stress and PMS [28]. Additionally, PA might still affect sex hormone function by modulating target tissue sensitivity to these hormones [29]. A previous study showed a significant decrease in prolactin, oestradiol, and progesterone levels, resulting in fatigue, impaired concentration, confusion, and most PMS symptoms. In addition, a study showed that increased prolactin level in the late luteal phase was one of the causes of breast pain and swelling. PA in non-athletes possibly reduces the status of this hormone; thus, the ratio of breast pain and swelling may be reduced. However, our results did not corroborate with this finding [30]. PA, including resistive exercise, induces neuroimmunomodulation effects, increases neurotrophies [31] and $\beta$-endorphins[32], decreases the sympathetic response, affects the hypothalamic-pituitary-adrenal axis reactions, and improves the serotonin system. All of these responses may decrease anxiety and depression [33].

Acne is a symptom associated with polycystic ovarian syndrome and is a common metabolic disturbance, particularly in women [34][35]. A study suggested that regular exercise decreased plasma insulin levels and reduced metabolic disease [36]. 
Sleep and activity during awake time interact to influence many aspects of health [37]. Moreover, a study showed that age and sex were associated with PA and sleep. They found that aspects of PA were significantly lower in younger adults (20-39 years old) who reported more frequent daytime sleepiness [38].

Priya et al. studied the effect of aerobic exercise at different intensities on PMS and concluded that moderate-intensity aerobic exercise should be encouraged as a potential prophylaxis for PMS [39]. This study gave us a range of PAs that they investigated. In this study, we converted different intensities of exercise into numbers that were calculated from the participants' daily PA. We were not simply considering exercise alone, but also the overall PA. In addition to high-intensity exercise in participants engaged in sports, we also examined the presence of daily moderate-intensity exercise and walking time.

In addition, we considered that PMS symptoms affected daily PA. A systematic review and meta-analysis indicated that exercise performance might be trivially reduced during the early follicular phase of the menstrual cycle compared with all other stages [18]. We considered the possibility that PMS symptoms affected exercise. Although some of the participants' PA included high-intensity exercise in our study, it could not be denied that PMS affected PA.

Thus, according to our results, women with 3000 METS/week or more of PA had milder symptoms of PMS.

\section{Study limitations}

Researchers frequently rely on questionnaires to assess PA because of their low cost and ease of administration. However, inherent limitations, such as participant recall bias and an inability to accurately retrospectively recall relevant PA details, may lead to overestimation or underestimation of PA. The target is limited, and causality is not known. The IPAQ-short version typically overestimates PA as measured by an objective criterion by an average of $84 \%$ [40].

\section{Conclusion}

This study is one of the few studies that analysed the relative factors of PMS based on regular PA using a questionnaire. The findings of this research showed that people with high PA had milder physical symptoms of PMS. This implies that recommending more regular PA as a non-pharmacological therapy is likely to be an effective treatment for PMS because it is a low-risk, low-cost, and simple method of symptom management for PMS.

\section{Abbreviations}

Premenstrual syndrome (PMS)

Premenstrual dysphoric disorder (PMDD)

Beats per minute (bpm)

Physical activity (PA)

International Physical Activity Questionnaire (IPAQ)

The Metabolic Equivalent of Task (MET)

Body Mass Index (BMI)

\section{Declarations}

\section{Data availability}


Data cannot be shared publicly because of the policy of the Kyoto University for data safety and protection. Data are available on request from the faculty of human health sciences for researchers who meet the criteria for access to confidential data or enrolled in the faculty. Data requests may be directed to the Kyoto University Graduate School and Faculty of Medicine, Ethics Committee, Department of Ethics Support (email: ethcom@kuhp.kyoto-u.ac.jp; telephone/fax: 0081-75-753-4680). Data requests may also be directed to Assistant Professor Momoko Tanima, who is in the department and unaffiliated with the current study (email: tanima.momoko.8s@kyoto-u.ac.jp; telephone: 0081-75-751-3964).

\section{Ethics approval and consent to participate}

Ethical approval was obtained from the Ethics Committee of Kyoto University Graduate School and Faculty of Medicine (No. R1442).

Informed consent was obtained from all individuals who participated in this study via an online questionnaire. Sending the questionnaire constituted consent to participate in this study, and all necessary explanations were provided in the online document. Participants under 20 years of age were asked to obtain consent from their parents.

\section{Consent for publication}

Not applicable.

\section{Competing interests}

The authors have no relevant financial or non-financial interests to disclose.

\section{Funding}

The authors declare that no funds, grants, or other support were received during the preparation of this manuscript.

\section{Authors' contributions}

SM designed the study, CYC collected data, analysed and interpreted the data, and wrote the manuscript. MT, RK interpreted the data and reviewed the manuscript. KM, MK, KS, HS, YS reviewed the manuscript. TA reviewed the manuscript and supervised the entire research process. All authors read and approved the final manuscript.

\section{Acknowledgement}

We would like to express our gratitude to all the participants who were very cooperative throughout the research period.

\section{References}

1. Chung S-H, Kim T-H, Lee H-H, Lee A, Jeon D-S, Park J, et al. Premenstrual Syndrome and Premenstrual Dysphoric Disorder in Perimenopausal Women. J. Menopausal Med. 2014;20:69, doi:10.6118/jmm.2014.20.2.69.

2. Halbreich U, Backstrom T, Eriksson E, O'Brien S, Calil H, Ceskova E, et al. Clinical diagnostic criteria for premenstrual syndrome and guidelines for their quantification for research studies. Gynecol. Endocrinol. 2007;23:123-30, doi:10.1080/09513590601167969. 
3. Angst J, Sellaro R, Stolar M, Merikangas KR, Endicott J.The epidemiology of perimenstrual psychological symptoms. Acta Psychiatr. Scand. 2001;104:110-6, doi:10.1034/j.1600-0447.2001.00412.x.

4. Yonkers KA, Simoni MK. Premenstrual disorders. Am. J. Obstet. Gynecol. 2018.

5. Ryu, A.; Kim, T.H.Premenstrual syndrome: A mini review. Maturitas 2015;82:436-40, doi:10.1016/j.maturitas.2015.08.010.

6. Dimmock PW, Wyatt KM, Jones PW, O'Brien PMS. Efficacy of selective serotonin-reuptake inhibitors in premenstrual syndrome: A systematic review. Lancet 2000;356:1131-6, doi:10.1016/S0140-6736(00)02754-9.

7. Pearce E, Jolly K, Jones LL, Matthewman G, Zanganeh M, Daley A. Exercise for premenstrual syndrome: A systematic review and meta-analysis of randomised controlled trials. BJGP Open 2020;4:1-11, doi:10.3399/bjgpopen20X101032.

8. Yoshimi K, Shiina M, Takeda T. Lifestyle factors associated with premenstrual syndrome: A cross-sectional study of Japanese high school students. J Pediatr Adolesc Gynecol. 2019;32:590-5, doi:10.1016/j.jpag.2019.09.001.

9. Maged AM, Abbassy AH, Sakr HRS, Elsawah H, Wagih $\mathrm{H}$, Ogila Al, et al. Effect of swimming exercise on premenstrual syndrome. Arch Gynecol Obstet. 2018;297:951-9, doi:10.1007/s00404-018-4664-1.

10. Dehnavi MZ, Jafarnejad F, Goghary SS.The effect of 8weeks aerobic exercise on severity of physical symptoms of premenstrual syndrome: A clinical trial study. BMC Womens Health 2018;18:1-7, doi:10.1186/s12905-018-0565-5.

11. Kamalifard M, Yavari A, Asghari-Jafarabadi M, Ghaffarilaleh G, Kasb-Khah A.The effect of yoga on women's premenstrual syndrome: A randomized controlled clinical trial. Int. J Women's Heal Reprod Sci. 2017;5:205-11, doi:10.15296/ijwhr.2017.37.

12. Ortiz MI, Cortés-Márquez SK, Romero-Quezada LC, Murguía-Cánovas G, Jaramillo-Díaz A P. Effect of a physiotherapy program in women with primary dysmenorrhea. Eur J Obstet Gynecol Reprod Biol. 2015;194:24-9, doi:10.1016/j.ejogrb.2015.08.008.

13. Ainsworth B, Cahalin L, Buman M, Ross R.The Current State of Physical Activity Assessment Tools. Prog Cardiovasc Dis. 2015;57:387-95, doi:10.1016/j.pcad.2014.10.005.

14. Kroll-Desrosiers AR, Ronnenberg AG, Zagarins SE, Houghton SC, Takashima-Uebelhoer BB, Bertone-Johnson ER. Recreational physical activity and premenstrual syndrome in young adult women: A cross-sectional study. PLoS One 2017;12:1-13, doi:10.1371/journal.pone.0169728.

15. Biernat E, Piątkowska M. Leisure time physical activity among employed and unemployed women in Poland. Hong Kong J Occup Ther. 2017;29:47-54, doi:10.1016/j.hkjot.2017.04.001.

16. Fernández-Bustos JG, Infantes-Paniagua Á, Cuevas R, Contreras OR. Effect of physical activity on self-concept: Theoretical model on the mediation of body image and physical self-concept in adolescents. Front. Psychol. 2019;10. doi:10.3389/fpsyg.2019.01537.

17. November R. Guidelines for Data Processing and Analysis of the International Physical Activity Questionnaire (IPAQ). 2005.

18. McNulty KL, Elliott-Sale KJ, Dolan E, Swinton PA, Ansdell P, Goodall S, et al. The effects of menstrual cycle phase on exercise performance in eumenorrheic women: A systematic review and meta-analysis. Sport Med. 2020;50:1813-27, doi:10.1007/s40279-020-01319-3.

19. Vishnupriya R, Rajarajeswaram P. Effects of aerobic exercise at different intensities in pre menstrual syndrome. J Obstet Gynecol 2011;61:675-82, doi:10.1007/s13224-011-0117-5.

20. Of M. Disorders, M. Diagnostic and Statistical Manual of Mental Disorders; ISBN 9780890425541.

21. Wong LP, Khoo EM. Menstrual-related attitudes and symptoms among multi-racial asian adolescent females.International journal of behavioral medicine 2011;246-53, doi:10.1007/s12529-010-9091-z.

22. Rapkin AA. Review of treatment of premenstrual syndrome \& premenstrual dysphoric disorder. Psychoneuroendocrinology 2003, 28 Suppl 3, 39-53. doi:10.1016/S0306-4530(03)00096-9.

23. Craig CL, Marshall AL, Sjöström M, Bauman AE, Booth ML, Ainsworth BE, et al. International physical activity questionnaire: 12-Country reliability and validity. Med Sci Sports Exerc. 2003;35:1381-95, doi:10.1249/01.MSS.0000078924.61453.FB.

24. Hashim MS, Obaideen AA, Jahrami HA, Radwan $\mathrm{H}$, Hamad HJ, Owais AA, et al. Premenstrual syndrome is associated with dietary and lifestyle behaviors among university students: A cross-sectional study from sharjah, UAE. Nutrients 2019;11:1-

Page 9/12 
18, doi:10.3390/nu11081939.

25. Jahromi KM, Gaeini A, Rahimi Z. Influence of a physical fitness course on menstrual cycle characteristics. Gynecol Endocrinol. 2008;24:659-62, doi:10.1080/09513590802342874.

26. Haskell WL, Lee IM, Pate RR, Powell KE, Blair SN, Franklin BA, et al. Physical activity and public health: Updated recommendation for adults from the American College of Sports Medicine and the American Heart Association. Circulation 2007;116:1081-93, doi:10.1161/CIRCULATIONAHA.107.185649.

27. Tremblay MS, Warburton DER, Janssen I, Paterson DH, Latimer AE, Rhodes RE, et al. New Canadian physical activity guidelines. Appl Physiol Nutr Metab. 2011;36:36-46, doi:10.1139/H11-009.

28. Wilmore JH, Costill DL, Gleim GW. Physiology of Sport and Exercise. Medicine \& Science in Sports \& Exercise; 1995;27;792.

29. Ennour-Idrissi K, Maunsell E, Diorio C. Effect of physical activity on sex hormones in women: A systematic review and metaanalysis of randomized controlled trials. Breast Cancer Res. 2015;17:1-11, doi:10.1186/s13058-015-0647-3.

30. El-Lithy A, El-Mazny A, Sabbour A, El-Deeb A. Effect of aerobic exercise on premenstrual symptoms, haematological and hormonal parameters in young women. J Obstet Gynaecol. (Lahore). 2015;35:389-92, doi:10.3109/01443615.2014.960823.

31. Eyre HA, Papps E, Baune BT. Treating depression and depression-like behavior with physical activity: An immune perspective. Front Psychiatry 2013;4:1-27, doi:10.3389/fpsyt.2013.00003.

32. Bodnar RJ, Klein GE. Endogenous opiates and behavior: 2005. Peptides 2006;27:3391-478, doi:10.1016/j.peptides.2006.07.011.

33. Lin TW, Kuo YM. Exercise benefits brain function: The monoamine connection. Brain Sci. 2013;3:39-53, doi:10.3390/brainsci3010039.

34. Hassan A, Gordon CM. Polycystic Ovary Syndrome in Adolescence. 2008, 1-8.

35. Michelmore KF, Balen AH, Dunger DB, Vessey MP. Polycystic ovaries and associated clinical and biochemical features in young women. Clin Endocrinol. (Oxf). 1999;51:779-786, doi:10.1046/j.1365-2265.1999.00886.x.

36. Mosca L, Grundy SM, Judelson D, King K, Limacher M, Oparil S, et al. Guide to Preventive Cardiology for Women.AHA/ACC Scientific Statement Consensus panel statement. Circulation 1999;99:2480-4.

37. Atkinson G, Davenne D. Relationships between sleep, physical activity and human health. Physiol. Behav. 2007;90:229-35, doi:10.1016/j.physbeh.2006.09.015.

38. McClain JJ, Lewin DS, Laposky AD, Kahle L, Berrigan D. Associations between physical activity, sedentary time, sleep duration and daytime sleepiness in US adults. Prev Med. (Baltim). 2014;66:68-73, doi:10.1016/j.ypmed.2014.06.003.

39. Vishnupriya R, Rajarajeswaram P. Effects of aerobic exercise at different intensities in pre menstrual syndrome. J Obstet Gynecol. 2011; 61(6):675-682 oi:10.1007/s13224-011-0117-5.

40. Lee PH, Macfarlane DJ, Lam TH, Stewart SM. Validity of the international physical activity questionnaire short form (IPAQSF): A systematic review. Int J Behav Nutr Phys Act. 2011;8:115, doi:10.1186/1479-5868-8-115.

\section{Figures}


In the past three menstrual cycles, at least one of the following physical symptoms or psychological

symptoms were observed during the 5 days before menstruation.

Psychological symptoms $\quad$ Physical symptoms
$\begin{aligned} & \text { Depression } \\ & \text { Anger }\end{aligned}$
Irritability
Anxiety
Confusion
Absent from society
symptoms were relieved within 4 days after the start of menstruation. They did not recur in the next 13
after the onset of menstruation.
symptoms were not due to drug therapy, hormonal internal use, drugs, or alcohol use.
symptoms must occur reproducibly during the two cycles of prospective recording.

\section{Figure 1}

Premenstrual syndrome (PMS) diagnostic criteria of the American College of Obstetrics and Gynecology (2000).

\begin{tabular}{|c|c|c|c|c|}
\hline & Symptoms & No symptoms & No problem but symptomatic & Problematic \\
\hline \multirow{10}{*}{ 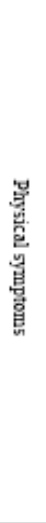 } & Breast tension and pain & $\square$ & $\square$ & $\square$ \\
\hline & Headache & $\square$ & $\square$ & $\square$ \\
\hline & Lower abdominal tightmess & $a$ & $\square$ & $\square$ \\
\hline & Lower abdominal pain & $\square$ & $\square$ & $\square$ \\
\hline & Swelling of hands and feet & $\square$ & a & $\square$ \\
\hline & Increased appetite & $\square$ & a & $\square$ \\
\hline & Easily fatigued and feeling listless & $\square$ & $\square$ & $a$ \\
\hline & Low back pain & $a$ & a & $\square$ \\
\hline & Sleepiness & $\square$ & $\square$ & $a$ \\
\hline & Acne & $\square$ & $\square$ & $\square$ \\
\hline \multirow{8}{*}{$\begin{array}{l}\text { 莺 } \\
\text { 总 } \\
\text { 总 } \\
\text { 思 }\end{array}$} & Feeling depressed & $\square$ & $\square$ & $a$ \\
\hline & Feeling irritable & $\square$ & $\square$ & 口 \\
\hline & Feeling confused & $\square$ & $\square$ & 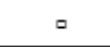 \\
\hline & Having feelings of sudden anger & $\square$ & $\square$ & $\square$ \\
\hline & Feeling anxious & $\square$ & $\square$ & a \\
\hline & A desire to be alone & a & $\square$ & a \\
\hline & Fatigue or lack of energy Overeating & $\square$ & $\square$ & $\square$ \\
\hline & Decreasing concentration & a & a & a \\
\hline
\end{tabular}


Figure 2

PMS symptoms questionnaire

A. In the majority of menstrual cycles, at least five symptoms must be present in the final week before the onset of menses. These symptoms should have started to improve within a few days after the onset of menses and should have improved or absent in the week after menses. B. One (or more) of the following symptoms must be present:

1. Marked affective lability (e.g., mood swings; feeling suddenly sad or tearful, or increased sensitivity to rejection).

2. Marked irritability or anger or increased interpersonal conflicts.

3. Marked depressed mood, feelings of hopelessness, or self-deprecating thoughts.

4. Marked anxiety, tension, and/or feelings of being keyed up or on edge.

C. One (or more) of the following symptoms must additionally be present, to reach a total of five symptoms when combined with symptoms from Criteria B, which can be seen above.

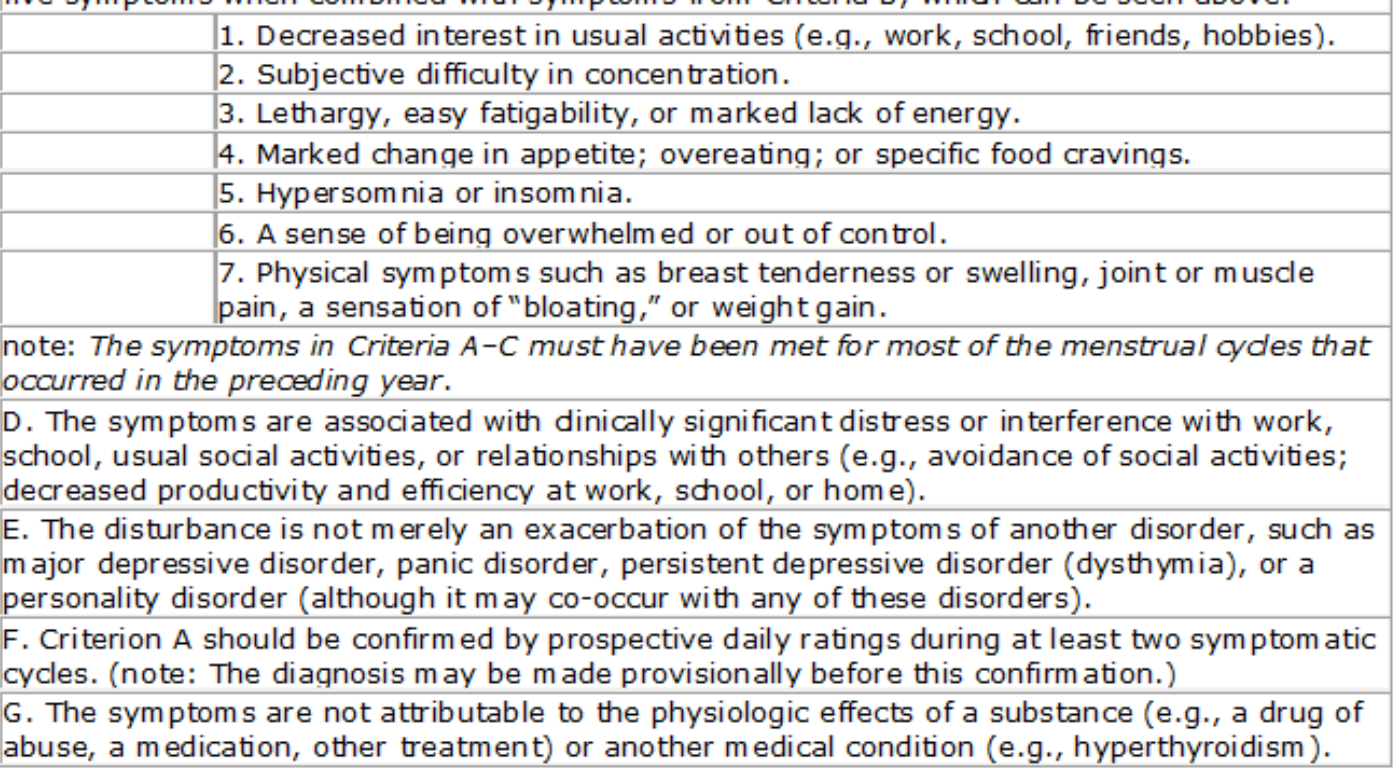

Reprinted with permission from the American Psychiatric Association. Diagnostic and Statistical

Manual of Mental Disorders. 5thed. Washington DC: American Psychiatric Association;

2013:171-172.

Figure 3

Diagnostic Criteria for Premenstrual Dysphoric Disorder

\section{Supplementary Files}

This is a list of supplementary files associated with this preprint. Click to download.

- Additionalfile.docx 